eral principles of physios and give the student a first-hand contact with the notions discussed in text-books, lectures and recitations. The emphasis is on the underlying ideas and the discussion of methods and accuracy of measurement is purely incidental. Books of this type are eminently suitable for students in elementary physics. On the other hand, the purpose of the manual may be to develop the theory and practise of physical measurements and to describe the construction and operation of standard measuring instruments. Such manuals are essential to the advanced student in physics and, if sufficiently comprehensive, they are useful to the student in chemistry or biology.

Professor Ferry's work belongs to the second category although a few of the experiments described would not be out of place in a manual of the first type. It is a thorough revision and rearrangement of an earlier book on "Practical Physics," by Ervin S. Ferry and Arthur T. Jones, to which chapters on sound, optics, electricity and magnetism have been added. The scope and method of the work are adequately indicated by the following quotations from the 'author's preface: "Only those experimental methods have been included that are strictly scientific and that can be depended upon to give good results in the hands of the average student. Although several pieces of apparatus, experimental methods and derivations of formulæ that possess some novelty appear, our fixed purpose has been to use the standard forms except in cases where an extended trial in large classes has demonstrated the superiority of the proposed innovation." "It has been assumed that the experiment is rare that should be performed before the student understands the theory involved and the derivation of the formula required. Consequently the theory of each experiment is given in detail and the required formula developed at length. The more important sources of error are pointed out, and means are indicated by which these errors may be minimized or accounted for."

Several of the methods of measurement described involve the use of instruments of spe- cial design not likely to be found outside of the author's laboratory but the greater part of them can be carried out with the apparatus that should be found in any well-equipped laboratory. The theory and manipulation of the more important modern instruments of precision are comprehensively treated and any student who has occasion to use such instruments will find these sections of the work very useful. The work is well adapted for use as a text in second- or third-year laboratory courses in physics. It should also find wide use as a reference book, in any laboratory where physical instruments and methods are occasionally used.

A. DEF. P.

\section{SPECIAL ARTICLES \\ NOTICE OF A RECENT CONTRIBUTION TO STATISTICAL METHODS}

Progress in science is measured, among other things, by the extent to which the qualitative treatment of problems is supplemented by a more rigorous quantitative treatment. The introduction of quantitative methods into the biological sciences, however, is beset with unusual difficulties. The highly complex and variable nature of the subject matter generally demands the empirical procedure of the statistician rather than the deductive one of the mathematician, and this is true of many problems of physical science as well, for example, those of meteorology. One of the main diffculties to be overcome arises from the simultaneous variation in the magnitudes of the many variables concerned. Especially is this true in "field" investigations where artificial control over the variable is impossible; as, for example, in marine ecology. In order to meet this difficulty the authors have prepared a paper entitled: "The functional relation of one variable to each of a number of correlated variables determined by a method of successive approximation to group averages." The introduction is written by Wm. E. Ritter under the title: "A step forward in the methodology of natural science."

1 Proc. Amer. Acadd. Arts. Sci., Vol. 55, Dee., 1919, pp. 89-133. 
This paper presents the development of a general method of ascertaining the relation between a dependent variable and each of a number of mutually correlated ones without being compelled to employ an assumed or predetermined mathematical function. This is accomplished by applying to the observed values of the dependent variable successive corrections based upon each value of all the independent variables. In this way is obtained a series of averages of the dependent variable corresponding to a series of averages of each one of the independent variables in turn and corrected to a constant value of each of the remaining ones. The method is concretely illustrated by an application to a bioclimatic problem; that of predicting the yield of South Dakota wheat from temperature and precipitation.

A limited number of reprints are available for distribution. Requests for them should be mailed to the Scripps Institution, La Jolla, California.

Geo. F. McEwen

Ellis L. Michael

\section{THE AMERICAN CHEMICAL SOCIETY VII}

Calorimetric determinations of the energy in yolk-protein and yolk-fat of doves and pigeons: OSCAR RIDDLES. Individual entire egg-yolks were separated into (boiling) alcohol-ether soluble and insoluble fractions. These moisture-free portions considered as yolk-protein and yolk-fat were burned in a Riche bomb calorimeter. Determinations were separately made upon yolks from various pure species and hybrids. The energy per gram of the yolk-protein of pure species averages 5,497 (small) ealories; for hybrids practically the same $(5,457)$. The energy per gram of yolk-fat of pure species averages 9,020 calories; for hybrids probably it is less $(8,897)$. The range of variability for yolks from individual hybrids is plainly greater than for yolks from pure species.

Some properties of the placental hormone: PAur M. GIesy. This substance, injected subcutaneously into the female animals, causes growth of the mammary glands and uterus. It was extracted by treating ground placentas with alcohol. In water, some, but not all, of the substance dissolves. It is dissolved by benzene, chloroform, carbon tetra- chloride, absolute alcohol, ether and ethyl acetate, but not by petroleum ether. If the extract is shaken with a mixture of water and benzene, the benzene solution alone is physiologically active. Alcoholic solutions and aqueous emulsions lose their activity on standing. The activity appears to be destroyed by continued heating.

The preparation of fatty acid esters of cholesterol: G. D. Beal aNd J. B. Brown. (By title.)

Comparative analysis of fibrin in the presence of various aldehydes: GroRgE E. Houm AND Ross AIKEN GORTNER. The comparative action of various amounts of paraldehyde, benzaldehyde, butyl and isobutyl aldehydes to that of formaldehyde when present in the acid hydrolysis of fibrin and gelatin was studied. In all cases the acid insoluble humin nitrogen increase is greater than with $\left(\mathrm{CH}_{2} \mathrm{O}\right)_{3}$ and a maximum is reached and maintained even in the presence of large excesses of these aldehydes. The ammonia nitrogen, soluble humin nitrogen and total amino nitrogen of the filtrates from the "humin" do not alter significantly. Using trioxymethylene, the increase in insoluble humin nitrogen is due to the presence of the indole nucleus, while with the other aldehydes tyrosin also enters into this reaction.

The preparation of cholesterol in quantity: PAUL M. GIESY. One hundred pounds of cattle spinal cords were ground, dehydrated with alcohol and extracted fourteen times with ether. The ether was evaporated from the extract, and the residue saponified by boiling with alcoholic sodium hydroxide. After evaporating the alcohol, the residue was taken up in water and extracted with ether. The ether was evaporated from the extract, and the cholesterol crystallized from alcohol. The first crop was cream-colored and melted at $147.1^{\circ}$ corrected. The second crop was brown, and melted at $146.4^{\circ}$. The total yield is about two pounds. The color can be removed by recrystallization from alcohol.

The infuence of aspartic acid and asparagin upon the eneymic hydrolysis of starch: H. C. SHERMaN AND Florence WaLker. (By title.)

An improved technic for measuring lipase activ. ity in animal or plant extracts or tissues: LERoY $\mathrm{S}$. PALMER (By title.) The material to be tested is added in the form of an extract or finely minced paste to at least 75 c.c. of artificial "'milk," prepared by grinding a suitable oil into hydrated acacia and diluting the emulsion with water. HCHO 1: 1,500 is added to the "'milk"' as preservtive. The initial acidity is determined by with- 